FERMILAB-TM-1740

\title{
Dijet Mass Resolution and Compensating Calorimetry
}

\author{
D. Green \\ Fermi National Accelerator Laboratory \\ P.O. Box 500 \\ Batavia, Illinois 60510
}

May 1991 


\section{DIJET MASS RESOLUTION AND COMPENSATING CALORIMETRY}

DAN GREEN

FERMILAB

\subsection{Introduction}

The calorimetry for SSC detectors has as its role the detection of the basic particles of the Standard Model. Those germane to calorimetry are quarks, photons, electrons, and gluons. Note that all the hadronic entities appear in the calorimetry as jets. The detection of single hadrons belongs to a past era when "quark molecules" were the focus of intense study. Thus, the goal of calorimetry at the SSC must be the study of jets. In particular, one must understand what defines the limits of accuracy of the jets. If there are intrinsic physical processes which limit the precision of jet measurements, then calorimetry which is more accurate is unnecessary if not wasteful.

\subsection{Dijet Mass Resolution}

The Monte Carlo study of dijets is a method whereby the intrinsic mass resolution may be determined. The question is, what determines the mass resolution? There are many contributions, and their affects will be considered separately. Details of the results are given in other papers for masses of $0.1 \mathrm{TeV}$ and $1.0 \mathrm{TeV}$ [1] and for 10.0 $\mathrm{TeV}[2]$.

\subsection{Contribution due to the Calorimeter}

The calorimeter error in determining energy, $\mathrm{dE}$, contributes to the mass error $\mathrm{dM}$. In what follows it is assumed that the angular error is unimportant. That is a question of the tower size and the total number of towers. The problem is "merely" economic and will not be considered further.

What about the jet? The limited transverse momentum of the fragmentation process means that the jet momentum is approximately the scalar sum of the momenta of the fragments. Assuming a calorimeter resolution consisting of a "stochastic" and a "constant" term for the individual hadrons, the jet momentum error can be found. The dijet mass resolution then follows under the assumption of low dijet transverse momentum, as shown in Eq. 1.

$$
\begin{aligned}
& P=k_{1}+k_{2}+k_{3}+\ldots \\
& d k / k=a / \sqrt{k} \oplus b \\
& d M / M \sim a / \sqrt{M} \oplus b<z 1>/ \sqrt{2}
\end{aligned}
$$

Note that the mass error, $\mathrm{dM}$, depends on the fragmentation process through the term proportional to $\langle z 1\rangle$, the mean fractional momentum of the "leading" fragment, $k i=z i P$. The jet momentum is $P$ and the fragment momentum is ki. At high masses, since the stochastic term falls as $1 / \sqrt{\mathrm{M}}$, the mass resolution is dominated by the constant term. To set the scale, if $a=0.5, b=0.03$ and $\langle z 1\rangle=0.2$, the stochastic contribution to $\mathrm{dM} / \mathrm{M}$ is $0.5 \%$ at $10.0 \mathrm{TeV}$ mass, while the constant term contributes $0.4 \%$ 


\subsection{Contribution due to Neutrinos}

The original jet may evolve into a $\overline{Q Q}$ pair. The hard $Q C D$ contribution is estimated to be proportional to the strong fine structure constant ( $\left.\alpha_{S}\right)$ and to increase logarithmically with the energy. A rough estimate of the resulting addition to the mass error is given in Eq. 2.

$$
\mathrm{d} M / M \sim \frac{1}{\sqrt{2}}\left[\left(2 \frac{\alpha_{S}}{\pi}\right) \ln (2 M Q / P) B(1 / 6)\right]
$$

The factor $B$ refers to the branching ratio for $Q-->$ qev. The factor $1 / 6$ is due to the fact that the $Q$ quark takes $\sim 1 / 2$ the momentum of the jet, and the neutrino takes $\sim 1 / 3$ of the $Q$ momentum. Given the soft logarithmic behavior, it is sufficient to take $M_{Q}=5$ $\mathrm{GeV}$, and the factor of 2 then takes $b$ and $c$ quarks into account. As an indication of the importance of neutrino "leakage", at $10.0 \mathrm{TeV}$ mass, the effect contributes $0.9 \%$ to $\mathrm{dM} / \mathrm{M}$. This contribution is comparable to that due to the calorimetry.

\subsection{Contribution due to Minbias Overlap}

The jet does not occur in a vacuum. It is embedded in the rest of the event. The energy flow of the event may be (under) estimated using the "minbias" events characteristic of the bulk of the inelastic interactions. Assuming a density of 8 particles per unit of rapidity, a mean kt per particle of $0.6 \mathrm{GeV}$, and a jet cluster cone of size $R=$ 0.6 , the minbias event has a transverse momentum of $\sim 1.1 \mathrm{GeV}$ accidentally falling within that cone. The event sets the scale of momentum fluctuations, Pfluc, which induces a term in the mass resolution;

$$
\mathrm{dM} / \mathrm{M}=\sqrt{2} \text { Pfluc } / \mathrm{M}
$$

At $M=10.0 \mathrm{TeV}$, the contribution is $0.015 \%$. The effect is only important at low masses.

\subsection{Contribution due to Fragmentation}

The soft jet fragmentation is characterized by a transverse momentum cutoff of $\sim \mathrm{kt}=0.6 \mathrm{GeV}$. Thus, there is an optimal cone size R. A large cone picks up most of the jet fragments but many "minbias" particles. A small cone picks up fewer jet fragments but also fewer "minbias" particles. The kt cutoff of the fragmentation, and the cone size determine a $k$ cutoff which defines a fluctuation momentum, Pfluc(sf), due to soft fragmentation.

$$
\begin{aligned}
& \text { Pfluc(sf) } \sim \mathrm{hkt} / \mathrm{R} \\
& D(z)=(h+1)(1-z)^{h / z}
\end{aligned}
$$

The simple form for the fragmentation distribution, $D(z)$, has $h=6$ [2], which means that Pfluc(sf) is $\sim 6 \mathrm{GeV}$ for a cone size $R=0.6$. At the $Z$ mass, $M=0.1 \mathrm{TeV}$, this error causes an error in $\mathrm{dM} / \mathrm{M}$ of $8.5 \%$. As this is near the quoted error for the $Z$ [1], it is anticipated that soft fragmentation dominates the error for low masses. 


\subsection{Contribution due to Gluon Radiation}

The Monte Carlo results for the mass error, $d M / M$, as a function of $R$ are shown in Fig. 1. There are no underlying event particles used in this Figure. Note that the falloff of $\mathrm{dM} / \mathrm{M}$ with $R$ is essentially the same for $M=0.1 \mathrm{TeV}$ and $M=10.0 \mathrm{TeV}$. $A$ glance at Eq. 3 and Eq. 4 indicates that soft fragmentation contributes a term which goes as $1 /(\mathrm{RM})$. It is expected [3] that the jets will shrink with mass. This particular behavior is not observed; $d M / M$ falls as $1 / R^{0.8}$ independent of $M$. The $1 / M$ behavior of soft fragmentation is not observed. For $M$ increasing by a factor of $100, \mathrm{dM} / \mathrm{M}$ decreases by only a factor $\sim 4$.

Some other fragmentation fluctuation must be at work. Consider the emission of a gluon by a quark in QCD. The energy difference between the initial and final states is,

$$
\Delta E=k(1-\cos (\theta))
$$

The gluon momentum is $k$ and the emission angle with respect to the quark is $\theta$. Since this "energy denominator" appears in the perturbative matrix element, one sees that the emitted gluons are preferentially soft, $\mathrm{k} \sim 0$, and collinear, $\theta \sim 0$. Nevertheless, there is a finite probability, $\mathcal{P}$, to emit a gluon with a momentum fraction $\mathrm{dP} / \mathrm{P}$ of the jet outside a cone defined by $R$ [3].

$$
P(R, d P / P) \sim\left(4 \alpha_{S} / 3 \pi\right)[4 \ln (R) \ln (2 d P / P)
$$

Equation 6 is an approximation to the expression given in Ref. 3 assuming that the logarithims are large. Note that this probability is independent of M. A plot of Eq. 6 is shown in Fig. 1 as the dashed line, assuming that $\mathrm{dP} / \mathrm{P}=\sqrt{2} \mathrm{dM} / \mathrm{M}$ and plotting a contour of constant (but arbitrary) probability. The shape appears to be quite similar to the observed dependence of $\mathrm{dM} / \mathrm{M}$ on $\mathrm{R}$.

A plot of the emission probability as a function of $\mathrm{dM} / \mathrm{M}$ for various cone sizes, $R$, is shown in Fig. 2 for $M=10 \mathrm{TeV}$. Note that $M$ enters implicitly in that $\alpha_{s}$ depends logarithmically on $M$. This dependence causes a slow logarithmic decrease of $\mathrm{dM} / \mathrm{M}$ at fixed R. The QCD scale , $\Lambda_{-}$, is taken to be $0.1 \mathrm{GeV}$. To set the scale, for a cone size of 0.3 , there is a $50 \%$ probability to radiate a single gluon which reduces $M$ by $1 \%$. Clearly, this effect is comparable to the others which have been discussed.

\section{$3.0 \mathrm{e} / \mathrm{h}$ and Mass Resolution}

\subsection{Total Dijet Mass Resolution}

The individual components discussed in Section 2 combine to yield the dijet mass resolution. Obviously, the contributions have a mass dependence. In Fig. 3 is shown the dijet mass resolution obtained by Monte Carlo methods [1], [2] as a function of $M$ for the range of $M$ which is relevant to the SSC. The lower limit is set by the $Z$ mass, while the upper limit is set by the rate limit due to the SSC luminosity. The individual contributions due to fragmentation, calorimeter resolution, and neutrino leakage are also shown. They were separated using Monte Carlo techniques.

The fragmentation at low mass is dominated by soft fragmentation, leading to $9 \% \mathrm{dM} / \mathrm{M}$ as was estimated previously. Since this factor falls as $1 / \mathrm{M}$, the fragmentation contribution is soon dominated, as $M$ increases, by the hard gluon 
emission, $M$ roughly $>1.0 \mathrm{TeV}$. The contribution of $\sim 2 \%$ at $R=0.6$ at $1.0 \mathrm{TeV}$ is, as $\mathrm{M}$ increases, subsequently quite a soft function of $M$, due to the logarithmic "running" of the coupling constant.

The fragmentation, hard and soft, dominates at all masses. The next most important factor is the resolution for masses $<3.0 \mathrm{TeV}$. The fact that it falls with $\mathrm{M}$ indicates that, Eq. 1, the stochastic term is the most important component of the calorimeter resolution. This occurs because the fragmentation is so soft that the individual particles making up the jet are fairly low energy.

Finally, at the highest masses the neutrino leakage term becomes important. For $M>3.0 \mathrm{TeV}$ the neutrino leakage is the second most important factor. Note that, since neutrino leakage rises with $M$, and hard fragmentation falls with $M$, eventually (but logarithmically) neutrino leakage will dominate the jet mass resolution.

\section{2 $\pi^{\mathrm{o}}$ Fluctuations, $\mathrm{e} / \mathrm{h}$, and Calorimeter Resolution}

The hadronic calorimeter which was used in the Monte Carlo had $a=0.5$, and $b=$ 0.03 (Eq. 1). It was assumed that the calorimetry responded to hadrons and photons equally. Suppose this is not the case and that the response is parametrized by a factor $\mathrm{e} / \mathrm{h}$ defining the difference in response. A given showering hadron bifurcates into a fraction of EM energy (fo) and a fraction (1 - fo) of hadronic energy. The combination of fluctuations in fo, dfo, and the nonuniform response, e/h $-1 \neq 0$, leads to energy fluctuations $\mathrm{dE}$.

Assume that dfo is due to statistical fluctuations in the number of neutral pions which are produced in the first interaction of a showering hadron (rather like the first dynode of a PMT). The mean number of neutral pions as a function of the incident hadron energy is shown in Fig. 4a. Note the roughly logarithmic rise with energy and the small number of neutrals. The small size of this mean implies a large fluctuation in fo.

$$
\begin{aligned}
& \mathrm{dfo}=\mathrm{fo} / \sqrt{\left\langle\mathrm{n}_{\pi 0}\right\rangle} \\
& \mathrm{dE} / E=|e / h-1| \mathrm{dfo}
\end{aligned}
$$

The value of dfo given by Eq. 7 is plotted as a function of energy in Fig. $4 \mathrm{~b}$. The value of dfo $=0.22$ [4] is called the noncompensation "constant term". In fact it is not constant, but falls with $E$ as the square root of the logarithm of $E$. This makes much more physical sense, since a shower of infinite momentum has so many produced pions that fo is stable. The form derived in Ref. 5 is also plotted in Fig. 4b. Obviously, the two curves are indistinguishable.

\section{$3.3 \mathrm{e} / \mathrm{h}$ and Mass Resolution}

Given the previous discussion, Eq. 1 and Section 3.2, one can estimate the effects of noncompensation. A response to hadrons and neutrals, $\mathrm{e} / \mathrm{h} \neq 1$, leads to a "constant term" $b(e / h)$. This is of order $6 \%$ for a very noncompensating calorimeter, $\mathrm{e} / \mathrm{h}=1.3$. This factor is then further reduced by the soft fragmentation of the jet into hadrons.

$$
\begin{gathered}
\mathrm{dM} / \mathrm{M}(\mathrm{e} / \mathrm{h}) \sim \mathrm{b}(\mathrm{e} / \mathrm{h})<\mathrm{z} 1>/ \sqrt{2} \\
\sim|\mathrm{e} / \mathrm{h}-1| \mathrm{dfo}<\mathrm{z} />/ \sqrt{2}
\end{gathered}
$$


The constant term of $6 \%$ is reduced to a $0.8 \%$ contribution to the dijet mass resolution, Eq. 8. A glance at Fig. 3 indicates that even a calorimeter with $\mathrm{e} / \mathrm{h}$ of 1.3 contributes only marginally to $\mathrm{dM} / \mathrm{M}$ for all the jet masses of interest to the SSC.

Using the parametrization advocated in Ref. 5, the effects of e/h were studied by Monte Carlo techniques. Since the effect of $e / h \neq 1$ is most important at the highest accessible masses, a $10 \mathrm{TeV}$ dijet was used. Results with both $\mathrm{e} / \mathrm{h}=1$ and $\mathrm{e} / \mathrm{h}=1.3$ were obtained. The reconstructed to generated mass ratio is shown in Fig. $5 \mathrm{a}$ and Fig. $5 \mathrm{~b}$ for $\mathrm{e} / \mathrm{h}=1$ and $\mathrm{e} / \mathrm{h}=1.3$ respectively. Clearly these results reinforce the previous conclusion that jet measurements are not compromised by a noncompensating calorimeter if $\mathrm{e} / \mathrm{h}<1.3$ and $\mathrm{M}<10.0 \mathrm{TeV}$. Note that high mass is the region of maximal sensitivity to $\mathrm{e} / \mathrm{h}$ (Fig. 3 ). The shift in $\mathrm{M}$ seen in Fig. $5 \mathrm{~b}$ is caused by the effective loss of hadronic energy. It is an artifact which can easily be removed by calibration.

\subsection{Summary}

Thus, although it is true that noncompensation is very important in the measurement of individual hadrons, the measurement of the fundamental particles which exist as asymptotic states only as jets is rather less sensitive. In fact, there are other physical processes which overshadow noncompensation (hard gluon emission, neutrino leakage) at all mass scales accessible to the SSC. Since the conclusion is independent of any physical process and simply relies on the fact that quarks/gluons are only observable as jets, one may hope that it is stable.

References

1. Letter of Intent by the Solenoidal Detector Collaboration, 30 November 1990 , SDC-70-00151

2. Depth Requirements in SSC Calorimeters, the FNAL SDC Simulation Group, to be submitted to Nuc. Inst. Meth.

3. G. Sterman and S. Weinberg, Phys. Rev. Lett., 39 23, 1436 (1977)

4. R. Wigmans, Nuc. Inst. Meth., A259, 389, (1987)

5. D. Groom, SSC-227, (1989)

\section{Figure Captions}

1. The fractional dijet mass resolution, $d M / M$, as a function of the clustering cone radius $R$. The points, 0 , refer to $0.09 \mathrm{TeV}$ dijets, while the points, $\bullet$, refer to 10 $\mathrm{TeV}$ mass. The solid lines are an eyeball fit of the form $\mathrm{dM} / \mathrm{M} \sim 1 / \mathrm{R}^{0.8}$. The dashed line is the form given in Ref. 3. for fixed probability $=0.6$. The points $\nabla$ are calculated using the formula from Ref. 3 .

2. The probability for a dijet to have a fractional mass $d M / M$ outside a cone of radius $R$ as a function of $d M / M$. The solid, dashed, and dot-dashed lines refer to $R=0.1$, 0.2 and 0.3 respectively.

3. The fractional mass resolution for dijets as a function of dijet mass $M$. The points are the results of a Monte Carlo calculation. The solid, dashed, and dot-dashed 
lines refer to the individual contributions to the resolution due to fragmentation, calorimeter resolution, and neutrinos from heavy quark decays respectively.

4. a. The mean number of neutral pions produced in an hadronic interaction as a function of the hadron energy.

b. The fluctuation in the fraction of energy in a hadron shower taken by neutral particles as a function of energy. The solid curve assumes that dfo is given by the statistical error in the number of neutral pions. The dashed curve is the form given in Ref. 5 .

5. a. The distribution of the ratio of generated to reconstructed dijet mass for 10 TeV dijets with default resolution.

b. As in a. save that the $\mathrm{e} / \mathrm{h}$ ratio is taken to be 1.3. 


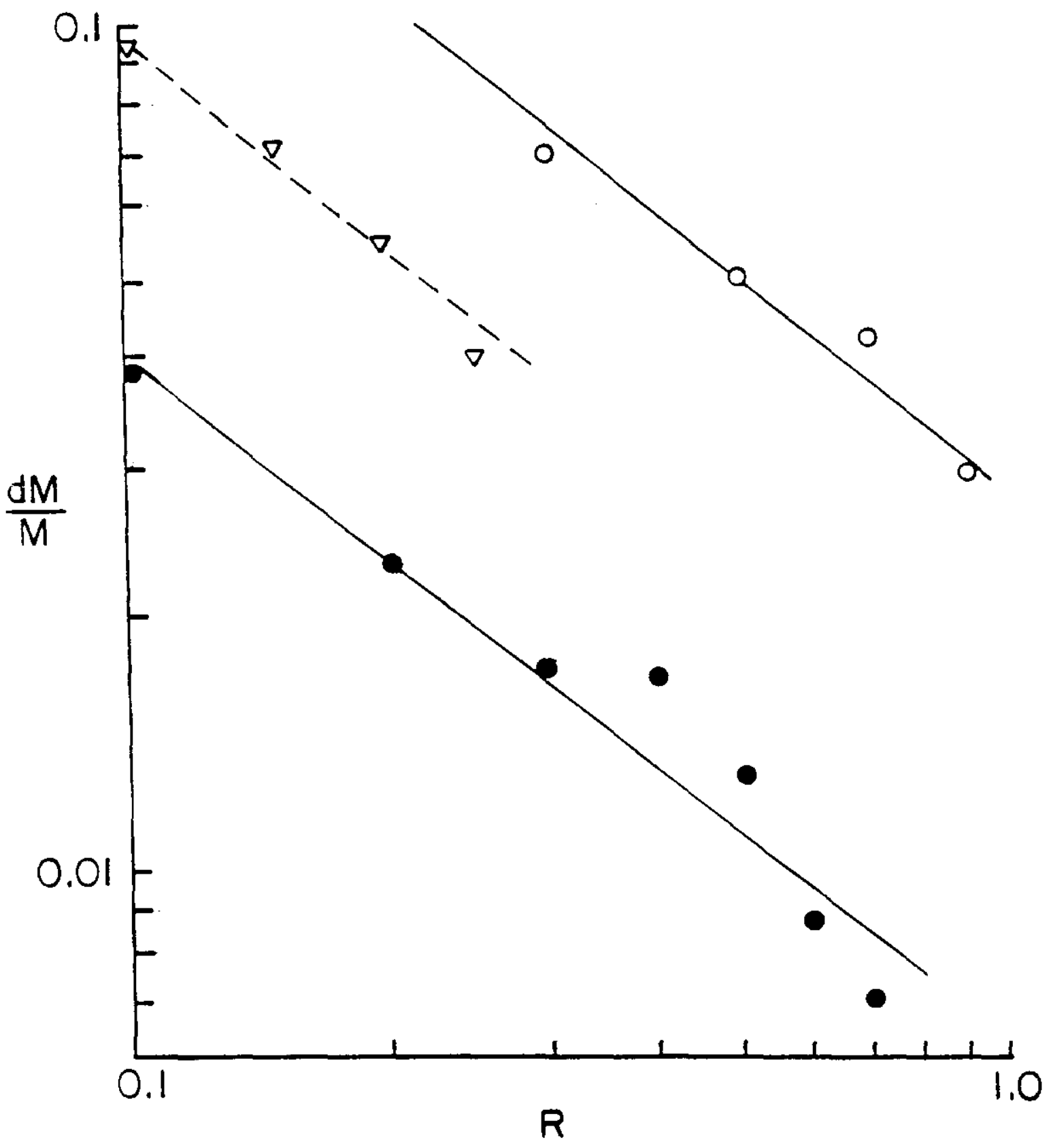

FIG. 1 


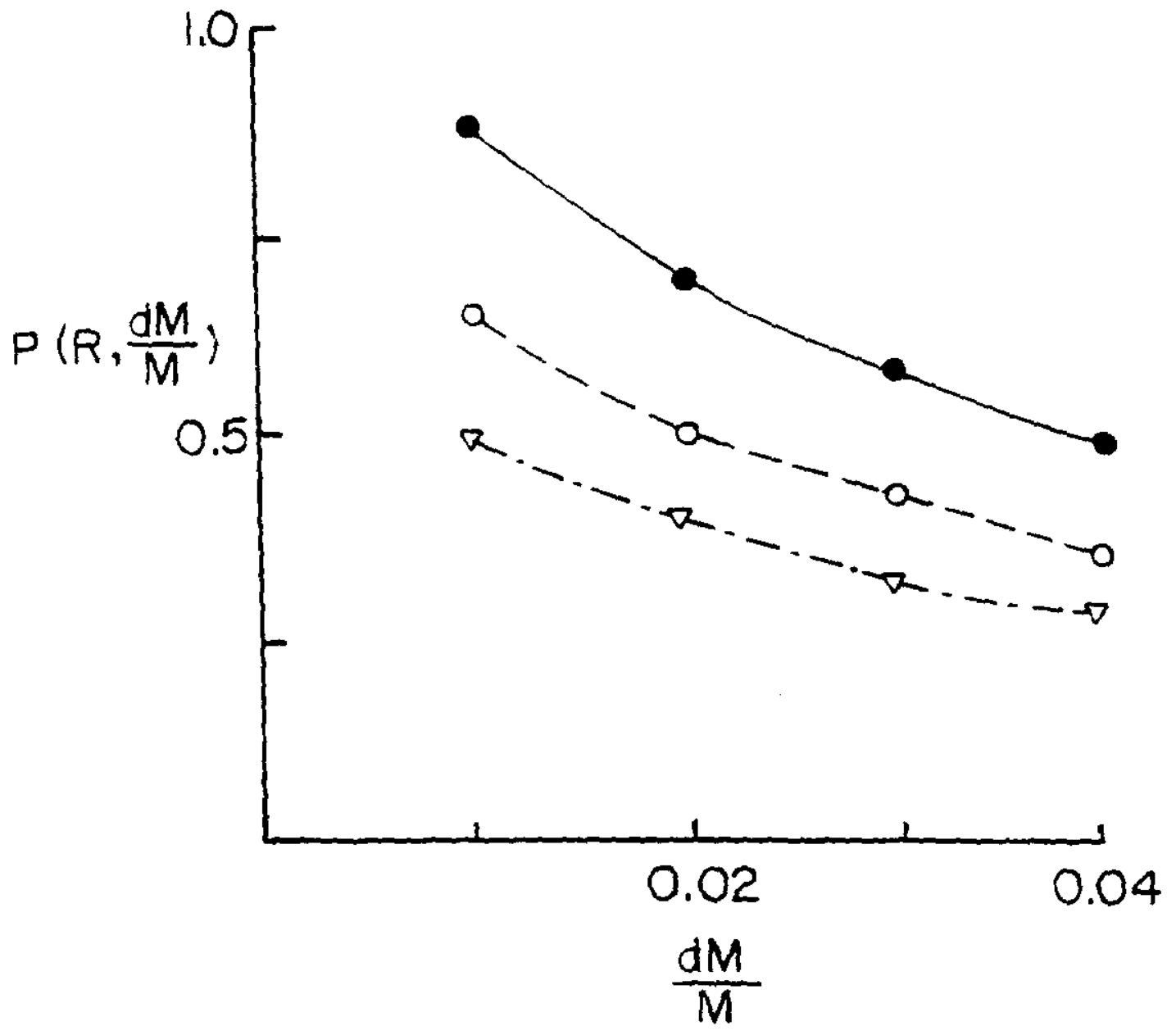

FIG. 2 


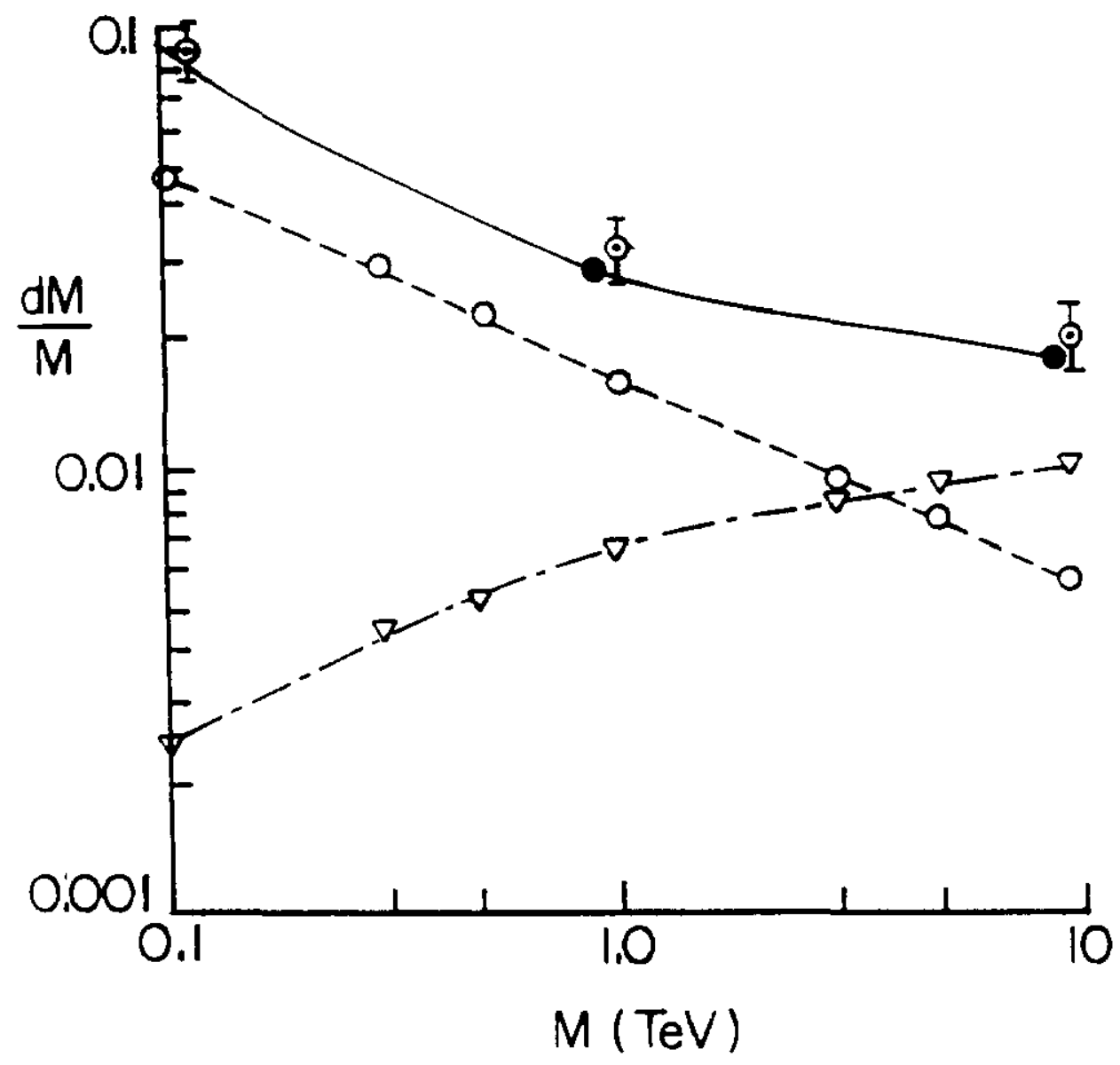

FIG. 3 


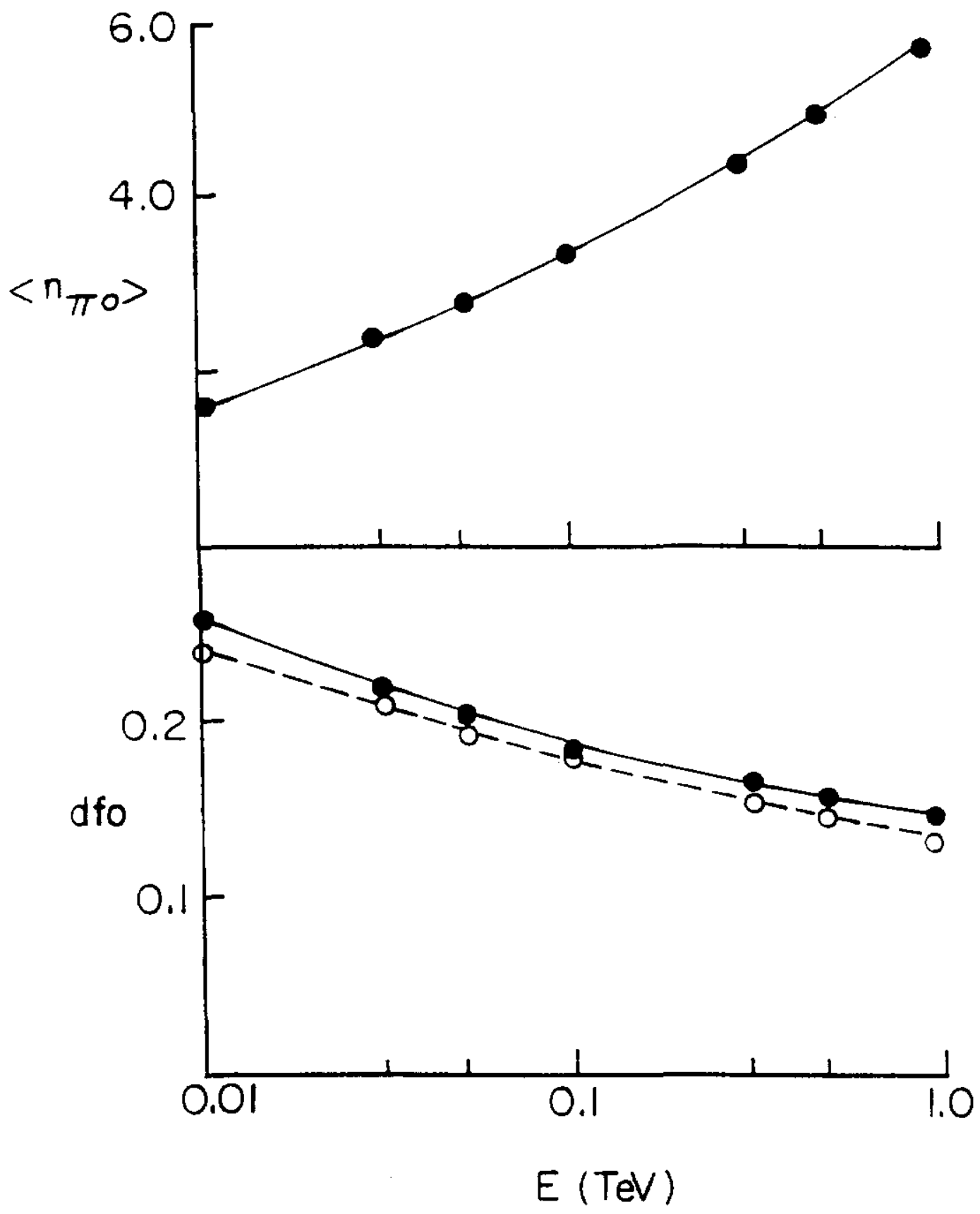

FIG. 4 

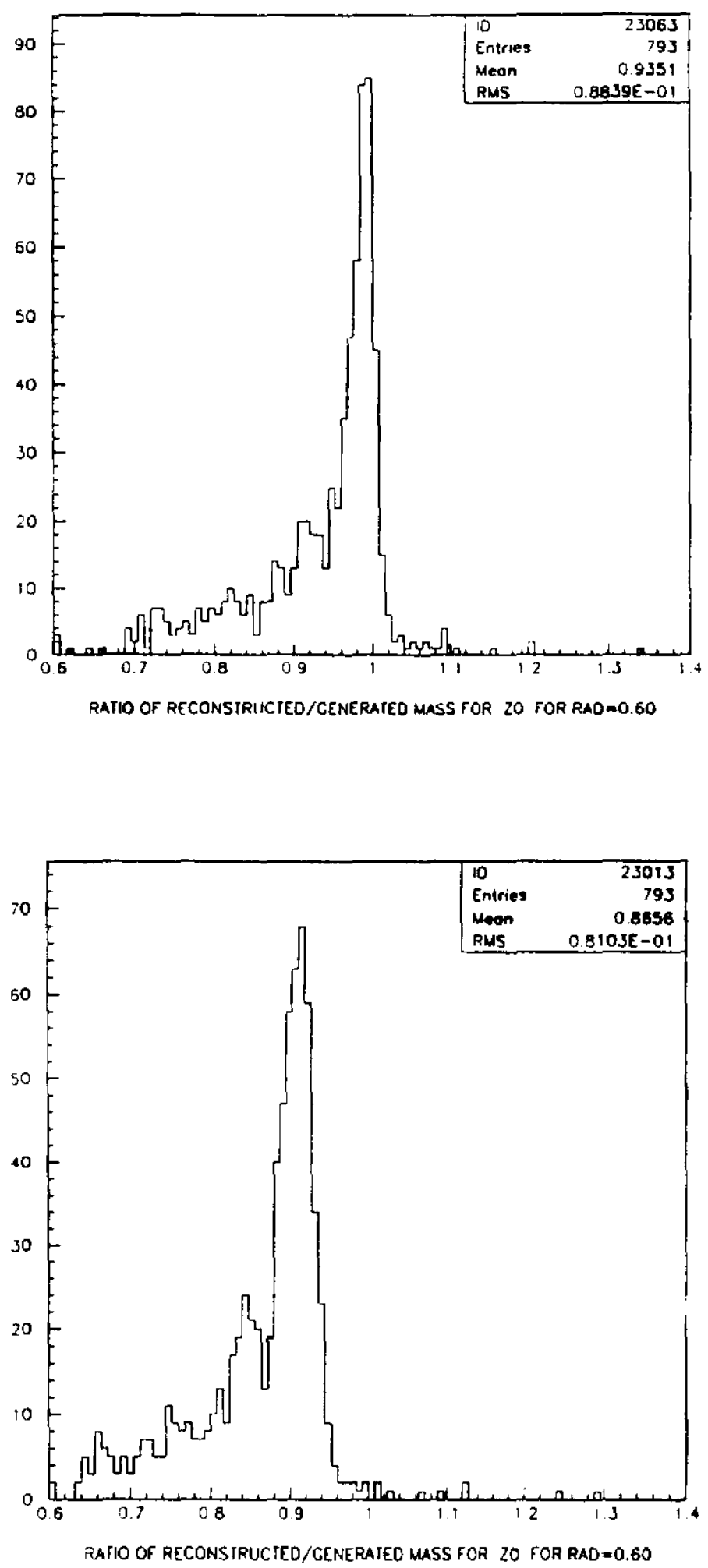

FIG. 5 